\title{
Robust Transcriptional Regulatory Response Upon Blocking NHEJ
}

Ronald Benjamin ( $\nabla$ ronald.babu@unlv.edu )

University of Nevada, Las Vegas

Atoshi Banerjee

University of Nevada, Las Vegas

Corey Guerunk

University of Nevada, Las Vegas

Lindsay Buczek

University of Nevada, Las Vegas

Danielle Eames

University of Nevada, Las Vegas

Sara Trimidal

University of Nevada, Las Vegas

Martin Schiller

University of Nevada, Las Vegas

\section{Research Article}

Keywords: DNA repair, TALEN, NHEJ, XRCC4(-/-), Mirin

Posted Date: April 30th, 2021

DOI: https://doi.org/10.21203/rs.3.rs-459760/v1

License: (c) (i) This work is licensed under a Creative Commons Attribution 4.0 International License.

Read Full License 
1 Robust transcriptional regulatory response upon blocking NHEJ

2 Ronald Benjamin $^{1 *}$, Atoshi Banerjee ${ }^{1}$, Corey Guerunk ${ }^{1}$, Lindsay Buczek ${ }^{1}$, Danielle Eames ${ }^{1}$, Sara

3 Trimidal ${ }^{1}$, and Martin R. Schiller ${ }^{*}$

4

$5 \quad{ }^{1}$ Nevada Institute of Personalized Medicine and School of Life Science ${ }^{2}$, University of Nevada

6 Las Vegas, Las Vegas, Nevada, 89154-4004 United States of America

7

8 Corresponding authors: Martin R. Schiller and Ronald Benjamin

9

10 Keywords

11 DNA repair, TALEN, NHEJ, XRCC4(-/-), Mirin.

12

13

14

15

16

17

18

19

20

21

22

23

24 


\section{Abstract:}

26 Double strand breaks are one of the most lethal forms of DNA lesions that, if left unrepaired can

27 lead to genomic instability, cellular transformation, and cell death. However, cells have two main

28 machineries namely error prone Non homologous end joining repair (NHEJ) or an accurate

29 homology dependent repair to repair the double strand breaks. NHEJ is the preferred mechanism

30 for DNA repair and basically consists of two forms: Canonical (C-NHEJ) and Alternative (A-

31 NHEJ) NHEJ. Our study examined the cellular repair outcome when NHEJ is blocked by targeting

32 two key DNA repair proteins: XRCC4 and MRE-11. We developed an extrachromosomal NHEJ

33 fluorescent reporter assay that uses Transcription activator-like effector nucleases (TALEN) to

34 introduce double strand breaks and detect the NHEJ editing by the presence of GFP fluorescence.

35 We demonstrated the presence of NHEJ editing in the XRCC4(-/-) cells treated with Mirin (a

36 pharmacological inhibitor of MRE-11), albeit with a $\sim 52 \%$ efficiency of the normal cells. The

37 transcriptional profiles of the Mirin treated HeLa XRCC4(-/-) cells had 307 uniquely differentially

38 expressed genes that was far greater than HeLa XRCC4(-/-) sample (83 genes) and Mirin treated

39 HeLa cells (30 genes). Pathway analysis unique to the XRCC4(-/-) +Mirin group included

40 differential expression of p53 downstream pathways, and metabolic pathways indicating cell

41 adaptation for energy regulation and stress response. In conclusion, our study showed that the

42 double strand DNA repair can be sustained even in absence of key DNA repair proteins XRCC4

43 and MRE-11. 
Introduction:

DNA carries genetic instructions for the development and function of all known living organisms;

51 therefore, it is important to preserve the integrity of the DNA. However, DNA is not inert, but

52 susceptible to multiple types of damages. The common sources of DNA damage include

53 environmental agents such as UV light, ionizing radiation, and chemical mutagens. Additionally,

54 endogenous biological processes such as cellular metabolism including oxidative damage, DNA

55 alkylation or hydrolysis, and double-strand breaks (DSBs) from collapsed replication forks

56 contributes to DNA damage ${ }^{1}$. In fact every day, DNA in normal cells has approximately 10,000

57 DNA aberrations and thus require an efficient repair of DNA damage to maintain its integrity ${ }^{2,3}$.

58 Failure to repair such damages can lead to genomic instability, cellular transformation, and cell

59 death.

60

61 To mitigate DNA damage and maintain integrity, cells have multiple molecular mechanisms to

62 repair different types of damages. The most deleterious DNA damage is arguably a DSB which, if

63 left unrepaired, threatens the loss of chromosomal content. The key machinery to repair DSBs are

64 (i) Non homologous end joining (NHEJ) repair and (ii) homology dependent repair (HDR) ${ }^{4}$. NHEJ

65 being the predominant form of repair, can commence in any phase of the cell cycle, unlike HDR,

66 which is active during the late $\mathrm{S}$ or G2 phase and is a less prevalent form of repair.

68 A mammalian cell can execute NHEJ repair within approximately $30 \mathrm{~min} 5$, although the

69 disadvantage is that it often introduces indels ${ }^{6}$. The most common form of NHEJ repair is

70 Canonical-NHEJ; catalyzed by two core protein-DNA complexes, Ku70/Ku80/DNA-PKC and

71 DNALig4/XRCC4/XLF-1 ${ }^{7}$. A backup repair pathway called Alternative-NHEJ (A-NHEJ) was

72 identified from persistent NHEJ activity in cells deficient for C-NHEJ ${ }^{8}$. A-NHEJ is evolutionarily 
conserved and can act in both C-NHEJ proficient or deficient cells ${ }^{9}$. A-NHEJ can mediate repair

74 with short homology at the site of DNA breaks and is also referred as microhomology dependent

75 DNA repair (MMEJ), although microhomology is not essential ${ }^{7}$.

77 Our earlier in silico analysis showed that the gene editing enzymes likely interfere with binding of 78 key components of DNA repair complexes and may alter the preference for C-NHEJ or A-NHEJ $79{ }^{10}$. Although XRCC4 and MRE-11 are well studied NHEJ complex proteins, we investigated 80 changes in cellular responses when both C-NHEJ and A-NHEJ are blocked by targeting these 81 proteins. Targeted inhibition of XRCC4, a component of the NHEJ ligation complex should block

82 NHEJ-mediated DNA repair. Schulte-Uentrop et al. demonstrated that knockout of XRCC4, a 83 component of the LIG4/XLF-1/XRCC4 complex renders cells sensitive to DNA damage caused 84 by either ionizing radiation or enzymatic cleavage of genomic DNA ${ }^{11}$. On the other hand, there is 85 a significant evidence supporting the role of MRN complex consisting of three proteins: meiotic recombination 11 protein (MRE-11), RAD50, and Nijmegen breakage syndrome 1 (NBS1; also

87 known as nibrin), in both C-NHEJ and A-NHEJ double strand break repair. The MRN complex is 88 also widely recognized for the role in HDR ${ }^{12-15}$. Depletion of MRE-11 is associated with the 89 reduction in the microhomology based repair in normal cells and inhibits resection in HeLa 90 XRCC4(-/-) cells supporting some role in both C-NHEJ and A-NHEJ ${ }^{16}$.

92 Using an NHEJ reporter construct, we show that NHEJ is prevalent even when both XRCC4 and 93 MRE-11 are blocked separately or simultaneously. Furthermore, the transcriptional profiles of the 94 HeLa XRCC4(-/-) cells treated with Mirin, a pharmacological inhibitor of MRE-11 had 307 95 uniquely differential expressed genes, much higher than the HeLa-NT (control), XRCC4(-/-) HeLa 96 and Mirin treated HeLa cells. The inhibition of XRCC4 and MRE-11 was associated with 
97 differential expression of p53 downstream pathways, and metabolic pathways indicating

98 adaptation of the cells to target regulation of energy and stress upon NHEJ inhibition. Our

99 experiments are the first to investigate broad cellular response when NHEJ is inhibited.

100

101 Materials and methods

102 NHEJ reporter plasmid construction

103 For construction of an NHEJ reporter plasmid, the sequence corresponding to a TALEN binding

104 site (Fig. 1A) was PCR amplified from pLai.2 HIV proviral plasmid (NIH AIDS Reagent Program

105 \#2532 ) and cloned upstream of the mCherry coding sequence into the NheI and AgeI sites of the

106 pmCherry-C1 plasmid (Takara \#632524). For cloning a TALEN binding site (TBS) downstream

107 of the mCherry coding sequence, the TBS was amplified, fused with GFP coding sequence by

108 overlap-PCR and cloned between the SalI and BamHI restriction enzyme sites. The coding

109 sequence of EGFP was amplified from the plasmid pEGFP-C3. A spacer was introduced between

110 the cytomegalovirus promoter (CMV) promoter and the upstream TBS at the NheI restriction

111 enzyme site (New England Biolabs) within the pmCherry-C1. Primers used for sequencing and

112 cloning are listed in (Supplementary Table 1).

\section{Cell culture and Lentivirus production}

115 HeLa (ARP 154) cells obtained from the NIH AIDS reagent program and LentiX293T cells

116 (Clonetech\# 632180) were grown in Dulbecco modified Eagle's Medium (DMEM) supplemented

117 with 10\% FetalClone III Serum (HyClone\# SH30109.03). Lentivirus was produced from

118 transfected LentiX293T cells. Briefly, 6 well plates were seeded with 0.6 million cells and

119 incubated for $24 \mathrm{hrs}$ prior to transfection. Cells were co-transfected with gXRCC4-

120 lenticrisprV2/gScrambled-lenticrisprV2, $1.2 \mu \mathrm{g}$; pspax-2 (Addgene. \# 12260), $1.0 \mu \mathrm{g}$, and pVSVG 
121 (Addgene. \# 22501), $0.3 \mu \mathrm{g}$ using the Lipofectamine LTX transfection reagent (Invitrogen) at a

122 1:3 ratio $[\mathrm{DNA}(\mu \mathrm{g})$ : Transfection reagent $(\mu \mathrm{l})]$. After $6 \mathrm{hrs}$ of incubation, media was replaced, and

123 cells were cultured in complete media for 48 hours. Cell supernatant were collected, filtered

124 through a $0.45 \mu \mathrm{m}$ syringe filter (Millipore), and used for transduction into HeLa cells.

125

126 Assessment of NHEJ repair

127 TALEN expression constructs (TAL 256 and TAL 278) were previously constructed with the 128 Joung Lab REAL Assembly TALEN kit ${ }^{17,18}$. To assess DNA repair, HeLa cells (0.6 million) were 129 seeded a day before transfection, co-transfected with a NHEJ reporter plasmid and pairs of TALEN 130 expressing constructs [TAL $256(200 \mathrm{ng})$ and TAL $278(200 \mathrm{ng})]$ at a ratio of 1:3 [DNA( $\mu \mathrm{g})$ :

131 Transfection reagent $(\mu \mathrm{l})$ ] with Viafect transfection reagent (Promega). As a control, NHEJ reporter 132 plasmid was co-transfected with empty vector [JDS70 (200ng) and JDS 78 (200ng)]. Media was 133 changed after 4 hrs incubation and replaced with complete media with or without Mirin (Sigma, $134100 \mu \mathrm{M}$ ). After 48 hours, cells were tested for mCherry and GFP fluorescence by fluorescence 135 microscopy and flow cytometry.

137 Generation of a XRCC4 knockout cell line - HeLa XRCC4(-/-)

138 To generate a XRCC4(-/-) HeLa cell line, a gXRCC4 sequence targeting the XRCC4 gene (NCBI 139 RefSeq: NC_000005.10) was annealed, phosphorylated with T4 Polynucleotide Kinase (New 140 England Biolabs) and cloned into the BsmBI digested LenticrisprV2 plasmid (Addgene \# 52961)).

141 The plasmid was delivered into HeLa cells by lentiviral transduction. The cells were selected in 142 complete media with puromycin $(1.5 \mu \mathrm{g} / \mathrm{ml})$ for 1 week followed by clonal selection. Clonal cells 143 were screened for biallelic XRCC4 knockout by Western Blotting with a XRCC4 antibody (Santa 144 Cruz Biotechnology sc-271087) on Nitrocellulose membrane (GE Amersham) and by targeted 
145 sequencing of gDNA. One of the clones 2G3 (HeLa XRCC4(-/-)) was selected for all subsequent

146 experiments. For the control HeLa cells, non-targeting guide RNA was similarly expressed in

147 HeLa cells. Genomic DNA (gDNA) was isolated from HeLa XRCC4(-/-) and HeLa-NT (control 148 cell containing non-targeting gRNA) using Quick DNA plus Kit (Zymogen). Isolated gDNA

149 spanning the selected region of the XRCC4 gene (NCBI RefSeq: NC_000005.10 (83104920.150 83105621)) was amplified by PCR with XRCC4-SeqV2FP and RA-XRCC4seq-RP primers and 151 Herculase II Fusion DNA polymerase (Agilent). PCR amplified product was digested with XhoI 152 (New England Biolabs), and subcloned into the XhoI and EcoRV restriction enzyme sites of the 153 pBlueScript II SK (-) vector. Several colonies were screened for the XRCC4 gDNA insert, and $154 X R C C 4$ knockout for the $2 \mathrm{G} 3$ clonal cells were confirmed by sanger sequencing.

\section{MTT assay for metabolic activity}

157 Cells were analysed with a 3-(4,5-dimethylthiazol-2-yl)-2,5-diphenyltetrazolium bromide (MTT) 158 chromogenic metabolic activity assay. HeLa-NT and XRCC4(-/-) cells were cultured in 96-well 159 plates for one day and an additional two days with complete media (DMEM+10\% FetalClone 160 SerumIII) with or without Mirin dissolved in Dimethyl sulfoxide (DMSO, $100 \mu \mathrm{M})$. DMSO

161 vehicle control was added to the control cells. Media was removed and $50 \mu$ of incomplete media 162 (DMEM) with $50 \mu \mathrm{MTT}(5 \mathrm{mg} / \mathrm{ml})$ was added to each well and incubated for $4 \mathrm{~h}$ at $37^{\circ} \mathrm{C}$ in a $163 \mathrm{CO}_{2}$ incubator. Resulting formazan crystals were dissolved with $100 \mu \mathrm{l}$ of acidified isopropanol, 164 incubated for 5 min at $37^{\circ} \mathrm{C}$, and absorbance at $595 \mathrm{~nm}$ was measured with a DTX 880 multimode 165 detector (Beckman Coulter).

167 Fluorescence Microscopy 
168 Transfected cells were cultured for 48 hours before measuring fluorescence. Live cells were scored

169 for EGFP and mCherry fluorescence in fresh media. Images were acquired at 20X magnification 170 with a Nikon TE2000E epifluorescence microscope equipped with Photometrics CoolSNAP FX 171 Camera (Roper Scientific). Images were captured in sequential scanning mode to avoid spectral

172 bleed through and were analysed in triplicates with multiple scanning regions for each.

173

174 Fluorescent Activated Cell Sorting (FACS)

175 Transfected cells were cultured for 48 hours before FACS analysis. Cells were trypsinized, washed 176 twice with phosphate buffered saline (PBS) and fixed with 2\% paraformaldehyde for 10 min at

177 RT. Cells were washed twice with PBS before acquisition with a flow cytometer (Sony SH800).

178 Cells were gated first for mCherry expression followed by GFP expression. Experiments were 179 analysed using FlowJo 10.7.1 for triplicate samples.

\section{$181 \quad$ RNA-seq and data processing}

182 Cells were transfected with a NHEJ reported plasmid and TALEN constructs and treated with 183 Mirin or vehicle control after 4 hrs post transfection. Cells were harvested $48 \mathrm{hrs}$ after transfection 184 and total RNA was extracted with the Zymogen RNA prep kit (Zymogen). Duplicate samples for 185 each condition were analyzed. RNA was quantified, RNA-seq was performed at a depth of more 186 than 20 million paired end reads for each sample (Novagene Corporation Inc).

188 The RNA-seq data can be accessed at Gene Expression Omnibus (GEO) (accession no 189 GSE135274). For RNA-seq analysis, raw reads were imported into CLC Genomics Workbench $190 \quad 12.0$ and trimmed using the quality limit score of 0.05 calculated from a modified-Mott trimming

191 algorithm ${ }^{19}$, read through adapter trimming, and trimming of ambiguous bases from read regions 
192 with more than two ambiguous reads ${ }^{19}$. RNA-Seq analysis was performed using default settings

193 including a mismatch cost of 2 with insertion and deletion cost of 3 . The Reference genome was

194 hg19 and reference gene track used was ensemble_v74. Differential expression analysis was

195 performed with the Identify and Annotate Differentially Expressed Genes (DEGs) software.

197 Bioinformatic analysis

198 A principal component analysis (PCA) of RNA-seq data and resulting plot were created with 199 ClustVis (https://biit.cs.ut.ee/clustvis/) ${ }^{20}$. Venn Diagrams of differentially expressed genes for 200 each condition was compared to control ( $\mathrm{FDR}<0.01 \log \mathrm{FC}>=1$ ) and plotted with an online tool 201 (http://bioinformatics.psb.ugent.be/webtools/Venn/). Heatmaps for the DEGs were plotted using 202 the Heatmap.2 function in the R ggplot package R (Warnes et al., 2020). Volcano plots for each 203 category were created using "EnhancedVolcano" function in R ${ }^{21}$. Gene enrichment analysis was 204 performed for DEGs lists $(\mathrm{FDR}<0.01$ and $\log \mathrm{FC}>1.2)$ with Metascape ${ }^{22}$. A network graph was 205 created with Cytoscape (v3.1.2). Term relationships having a similarity score above 0.3 are 206 connected by edges. The network is visualized with Cytoscape (v3.1.2).

\section{Protein-protein interaction (PPI) network analysis and pathways interrelation analysis}

209 PPI networks were constructed using multiple DEGs list based on BioGrid, InWeb_IN and

210 OmniPath database in Metascape. For networks which contain between 3-500 proteins, Molecular

211 Complex Detection (MCODE) algorithm was used to identify densely connected network

212 components with default parameters. For each MCODE component, pathway and process

213 enrichment analysis was applied and the three best-scoring (by $p$ value) terms were retained as the

214 functional description of the resulting modules. Resulting network graphs were visualized through

215 Cytoscape (v3.1.2). 


\section{Quantitative Real-time Polymerase Chain Reaction (qRT-PCR)}

218 For validation of RNA-seq results, cells were transfected with a NHEJ reported plasmid, TALEN

219 expression constructs, and treated with Mirin or vehicle control as described above. Cells were

220 harvested 48 hrs after transfection, Total RNA was extracted using the Zymogen RNA prep kit

221 (Zymogen) and cDNA was synthesized with SuperScript ${ }^{\mathrm{TM}}$ IV VILO ${ }^{\mathrm{TM}}$ Master Mix (Invitrogen).

222 Gene expression of select DEGs were quantified by qRT-PCR with gene-specific primers (Table

223 S1), and PowerUp SYBR Green PCRmix (Invitrogen) in a Bio-Rad CFX96 Touch ${ }^{\mathrm{TM}}$ Real-Time

224 PCR Detection System. Relative expression levels were normalized to a housekeeping control 225 gene. $\beta$-actin. The fold changes in mRNA levels between the HeLa-NT and the experimental 226 condition were calculated using the $2^{-(\Delta \Delta C T)}$ method.

\section{Statistical Analyses}

229 Statistical analyses were conducted with Student's $t$-test or by ANNOVA for comparing more than 2302 groups and a $p$-value $\leq 0.05$ was considered significant.

\section{Results}

\section{Construction and validation of a NHEJ reporter assay}

234 In order to test and detect NHEJ repair when TALEN introduces DSBs, we first constructed and

235 tested a plasmid encoding an extrachromosomal NHEJ reporter assay system. The reporter plasmid

236 has a CMV promoter for constitutive expression of the mCherry coding sequence. The mCherry

237 coding sequence is flanked on either side with TBSs (Fig. 1A). Under normal conditions, the 238 mCherry reading frame terminates with a stop codon. Consequently, the downstream GFP coding 239 sequence lacks a promoter; hence not expressed. When the cells are co-transfected with the 
240 reporter plasmid and the pair of TALEN expressing plasmids, that targets the encoded TBSs,

241 double strand breaks are introduced at both TBSs excising the mCherry coding region. Upon

242 subsequent NHEJ repair, the CMV promotor is ligated in proximity to the GFP coding region

243 driving its expression. Therefore, GFP is expressed upon NHEJ repair. A flow chart depicting the

244 relationships between DNA editing and fluorescence output is shown (Fig. 1A).

246 First, we tested the NHEJ reporter assay system in HeLa cells. The reporter plasmid was co-

247 transfected with TALEN expression constructs T256 and T278. GFP positive $\left(\mathrm{GFP}^{+}\right)$cells were

248 detected indicating editing by NHEJ (Fig. 1B). Co-transfection of the NHEJ reporter plasmid with

249 empty vectors, or with either one of the TALEN pair did not express any $\mathrm{GFP}^{+}$cells as expected

250 for cells that are not edited (Fig. 1B). This experiment confirms that our NHEJ assay system is

251 functional, specific, sensitive, and detects DNA repair.

\section{Inhibition of XRCC4 and MRE-11 in Hela cells}

254 To determine the effect of XRCC4 and MRE-11 on C-NHEJ and A-NHEJ repair pathways, we 255 needed to abolish expression of these proteins. XRCC4 was knocked out in Hela cells using 256 targeted CRISPR-Cas9 editing within the coding region. The XRCC4(-/-) biallelic knockout in 257 clone 2G3 was confirmed by the loss of XRCC4 protein expression as detected by Western blot 258 analysis (Fig. 1C). A complete absence of XRCC4 protein expression was observed, and this clone 259 was selected for further experiments. The knockout in clone $2 \mathrm{G} 3$ was confirmed by Sanger 260 sequencing, which revealed a $2 \mathrm{bp}$ deletion and $10 \mathrm{bp}$ deletion at both the alleles in the XRCC4 261 gene (Fig. 1D). MRE-11 expression was indispensable for cell survival thus, MRE-11 was 262 inhibited by Mirin, a well-established inhibitor of MRN complex and MRE-11 exonuclease 263 activity. We also analyzed the cell survival when $X R C C 4(-/-)$ cells were treated with Mirin (Fig. 
264 1E). Blocking both XRCC4 and MRE-11 indicate that that the cells are viable as detected in an 265 MTT assay (Fig. 1E).

NHEJ repair is sustained when XRCC4 and MRE-11 are blocked

268 Since cells with both XRCC4(-/-) and MRE-11 blocked survived and were metabolically active, 269 we next assessed the impact of blocking NHEJ on DNA repair activity. XRCC4(-/-) and control 270 cells HeLa-NT (Non-targeting) transfected with reporter plasmid and empty TALEN vectors did

271 not show any $\mathrm{GFP}^{+}$cells as expected for these negative controls (Fig. 2A \& E). Mirin treatment of 272 these cells also had no effect on GFP expression (Fig. 2B \& F). mCherry expression indicated that 273 the cells were expressing the reporter construct (Fig. 2). HeLa-NT and XRCC4(-/-) cells co274 transfected with the NHEJ reporter and TALEN constructs (T270 and T278) showed GFP 275 expression, indicating NHEJ repair (Fig. 2C \& G).

277 In co-transfected HeLa-NT cells treated with Mirin, $\mathrm{GFP}^{+}$cells were present indicating NHEJ 278 repair when MRE11 is inhibited (Fig. 2D). Surprisingly, co-transfected Mirin treated XRCC4(-/-) 279 also showed NHEJ repair as indicated by the presence of GFP expression (Fig $2 \mathrm{H}$ ). This suggests 280 that NHEJ repair is sustained even when XRCC4 and MRE-11 are inhibited.

282 NHEJ efficiency was lowered when both XRCC4 and MRE-11 are blocked

283 To quantify the NHEJ repair efficiency, cells expressing the fluorescent reporters were quantified 284 by flow cytometry. Cells were first gated based on mCherry ${ }^{+}$expression, selecting the population 285 expressing the reporter system. Next, we determined percentage of cells expressing GFP within 286 the mCherry ${ }^{+}$cell populations (Fig. 3A, B). The mean percentage of $\mathrm{GFP}^{+}$expressing cells within 287 the mCherry $^{+}$cells for each sample is compared in Fig. 3B. The efficiency of NHEJ repair was 
$288(25.5 \% \pm 1.3)$ in HeLa-NT cells and $(15.4 \% \pm 0.4)$ for HeLa-NT cells treated with Mirin (Fig. 3B),

289 indicating that blocking MRE11 activity reduced NHEJ editing efficiency. Similar inhibition of

290 NHEJ editing was observed in XRCC4(-/-) cells $(15.9 \% \pm 0.2)$ or those treated with Mirin $(13.26 \%$

$291 \pm 0.2)$. These results indicated that NHEJ repair was prevalent even when either or both XRCC4

292 and MRE-11 are blocked, however; the efficiency is reduced by approximately $40-48 \%$.

293

294 Altered expression of genes when NHEJ is blocked

295 The reporter assay demonstrates that NHEJ repair is partially inhibited, but still present in cells 296 when XRCC4 and MRE-11 expression is blocked alone, as well as in combination. To assess the 297 molecule basis for the robustness in preservation of NHEJ activity, gene expression of 298 transcriptomes was compared for cells with inhibition of XRCC4 and MRE-11. To determine 299 differences in transcriptomes, cell populations selected as above were analyzed by RNA-seq. A 300 principle component analysis showed a clear separation between each sample category, but 301 clustering for duplicate samples (Fig. 4A), indicating a different transcriptomic profile for each 302 sample category.

304 The transcriptional changes are summarized in a Venn diagram to codify similar and unique genes 305 among samples. The XRCC4(-/-) sample had 83 and Mirin treated HeLa cells had 30 unique 306 differentially expressed genes (Fig. 4B). However, the Mirin treated XRCC4(-/-) cells had 307 307 uniquely differentially expressed genes, far greater than other samples reflecting a more impactful 308 transcriptional response. This differential transcriptional response was further supported when the 309 top differentially expressed genes (DEGs) were plotted as heatmap (Fig.4C and Supplementary 310 Table 2). The gene expression for XRCC4(-/-) cells treated with Mirin were most different from 311 HeLa-NT cells (Fig. 4C). To validate the gene expression quantitation, five differentially 
312 expressed genes identified from the RNA-seq analysis (CA9, CDKN1A, ENO2, DUSP5 and

313 ZMAT3) were assessed by real time PCR. The top differentially expressed genes plotted for

314 heatmap (Fig. 4) and the p53 downstream pathway were selected for gene expression quantitation

315 by real time PCR (Fig. 5A). The PCR data and RNA-seq gene expression measurements were

316 consistent with each other, thereby validating the RNA-seq results (Supplementary Fig. 1). The

317 cells with either XRCC4 knock out or treated with Mirin showed fewer and less intense changes

318 in gene expression. Additional details for changes in gene expression are shown by volcano plots

319 in Supplementary Fig 2.

\section{Pathway analysis of differentially expressed genes (DEGs)}

322 We next sought to determine which pathways are functionally associated with the transcriptional

323 response to blocking NHEJ. DEG enrichment was analyzed with Metascape ${ }^{22}$. Meta-enrichment

324 analysis indicated that all 3 conditions with partially or completely blocked NHEJ were

325 consistently enriched in several pathways: transcriptional misregulation in cancer, alcoholism,

326 defense response to viruses, and response to oxygen levels (Fig. 5A). Pathways unique to XRCC4(-

327 /-) cells with or without Mirin were Extracellular matrix organization, core matrisome, and

328 endoderm and fat differentiation pathways (Fig. 5A). Pathways like carboxylic acid biosynthetic

329 process, p53 downstream pathways and HIF signaling pathway were enriched in XRCC4(-/-) +

330 Mirin group and in HeLa-NT treated with Mirin. Interestingly, pathways unique to XRCC4(-/-)

331 with Mirin group were regulation of small metabolic processes, TP53 regulates transcription of

332 cell death gene, generation of precursor metabolites and energy (Fig. 5A). A network layout was

333 created by enrichment analysis and visualized with Cytoscape (v3.1.2) (Fig. 5B). Such network

334 reveals interrelation of enriched pathways and genes. 


\section{PPI network construction and pathways interaction analysis}

337 Pathways were also evaluated by examining protein-protein interaction networks. A protein338 protein interaction network was constructed from the DEGs for the 3 sample conditions with 339 BioGrid in Metascape ${ }^{22}$. The network contained a total of 237 nodes and 956 edges where network 340 nodes are displayed as pies (Fig. 6A). Seven significant network modules were identified with the 341 MCODE algorithm (Fig. 6B), which included 71 proteins from which ENO3, CACNG6, ITGB8, 342 PDE10A, COL12A1 and FSTL3 served as seed proteins. Gene Ontology (GO) terms associated 343 with each module are depicted in Fig. 6B and HDAC deacetylates histones appear to be the 344 prominent GO term. As expected, human histone acetylation and deacetylation regulate the NHEJ 345 repair pathways ${ }^{23}$.

\section{DISCUSSION}

348 There are two NHEJ repair pathways for repairing DSBs upon DNA damage. We sought to better 349 understand the relationship between these pathways. First, we designed, tested, and validated a

350 NHEJ reporter construct that enabled us to examine the cells that have undergone NHEJ mediated 351 DNA repair. When XRCC4 is knocked out or MRE-11 exonuclease activity is inhibited, NHEJ 352 mediated DNA repair was reduced by $\sim 40 \%$. Interestingly, NHEJ activity was reduced to $\sim 50 \%$ 353 in XRCC4(-/-) + Mirin treated cells compared to wild type HeLa-NT cells. We predicted that 354 blocking NHEJ would increase vulnerability to cell death due to reduced ability to repair DNA 355 damage. This observation indicates that NHEJ is sustained, even when XRCC4 and MRE-11 and 356 presumably the functions of the know NHEJ pathways are abolished. These observations support 357 NHEJ pathways with redundant functions, that may be mediated by a transcriptional response. 358 
359 We considered three possible explanations for the presence of NHEJ repair despite inhibiting

360 XRCC4 and MRE-11: 1) There could be a yet to be discovered NHEJ pathway. However, this

361 possibility is unlikely considering that the comparative transcriptomic profiles did not reveal any

362 differential expression of DNA repair proteins or components of DNA repair complexes. We

363 recognize that this negative result is not conclusive, as is the case for any negative result. However,

364 analysis of whole transcriptome is a global measurement, but there could be genes with repair

365 functions that are not yet identified or annotated; 2) Another possible explanation is that either

366 MRE-11 or XRCC4 have other genes that can compensate for the lack of their functions. Although

367 Xing et al., identified $P A X X$, a new paralogue of $X R C C 4^{24}$, this gene was not differentially

368 expressed in our case; and 3). Another possibility, which we favor is that post translational

369 modifications of known DNA repair proteins or new protein-protein interactions modulate NHEJ

370 pathways and the changes in post-translational regulation would not be captured by the RNA-seq

371 analysis.

372

373 Considering the latter hypothesis, our DEG analysis identified multiple pathways that were 374 induced for each condition that block NHEJ. The result suggests induction of a substantial 375 regulatory response that may produce signaling cross talk between the NHEJ repair pathways.

376 Although, NHEJ is the major repair pathway for DSB; the temporal recruitment of different factors

377 and complexes are not completely elucidated. It is likely that MRE-11 may function upstream of 378 the XRCC4 involvement in the DNA repair and may suggest interaction between these NHEJ 379 pathways.

381 In support of cross-talk hypothesis, studies on cross talk of NHEJ with base excision repair (BER)

382 and HDR with some common proteins were identified ${ }^{25,26}$. Xia et al. has demonstrated that Pol $\beta$ 
383 which plays a central role in BER, exhibits higher degree of spatial colocalization with $\mathrm{Ku} 70$, a

384 component of C-NHEJ in the nucleus following DNA damage caused by Methyl methanesulfonate

385 or etoposide ${ }^{27}$. In vitro binding assays also supports interaction between Pol $\beta$ and Ku70. MRE-11

386 has a critical role in DSB recognition and protein complex recruitment in both the NHEJ and HR

387 pathways. Another protein, BRCA-1, is known to regulate the nuclease activity of MRE-11.

388 BRCA1 phosphorylation mediated by Checkpoint kinase-2- enhances NHEJ fidelity but can also

389 deplete 53BP in $\mathrm{S} / \mathrm{G} 2$ phase to favor $\mathrm{HR}$, suggesting that post-translation modification of BRCA1

390 regulates pathway preference ${ }^{25,26}$. Thus, it is possible that both C-NHEJ and A-NHEJ could also

391 have signaling interactions that rescue blocking both NHEJ pathways ${ }^{9,28}$.

393 One theme that emerged from blocking NHEJ was that blocking of NHEJ pathways by either

$394 X R C C 4$ knock out or Mirin treatment alone or in combination lead to pathways like transcriptional

395 misregulation in cancer, alcoholism, defense response to viruses, and response to oxygen levels.

396 The error prone nature of NHEJ is a major cause of carcinogenesis and blocking of these pathways

397 in our experiments correlates with the transcriptional misregulation associated with cancer

398 pathway. Introduction of DSB/viral DNA leads to activated DNA repair pathway components

399 which are also activated as a defense response to viruses ${ }^{29}$. Similarly, oxidative stress induces

400 DNA damage and activate NHEJ mediated pathways ${ }^{30}$. Enrichment of these pathways highlight

401 cellular adaptation in response to DNA damage.

402

403 Although, we did not identify any major DNA repair protein to be uniquely altered in double

404 inhibition of XRCC4 and MRE-11 in cells, a bypass pathway may utilize some proteins already

405 known to be associated with NHEJ. The pathways enriched in these cells include pathways

406 associated with regulation of small molecule metabolic processes, TP53 regulates transcription of 
407 cell death genes and generation of precursor metabolites and energy suggesting a major role played

408 by genes of metabolic pathway in such a scenario. The metabolic enzymes are known to play non-

409 canonical roles outside their established metabolic roles especially in gene regulation, DNA

410 damage response and apoptosis ${ }^{31}$. Among the metabolic pathway, genes belonging to glycolysis

411 were more prevalent and were downregulated. Induction of glycolysis contributes to enhancement

412 of NHEJ repair pathway and here we show that XRCC4(-/-) with Mirin treated cells had lower

413 expression of glycolytic genes, PGK1, ALDOC, PFKFB4, TPI1, ENO3, PFKP, ENO2, HK2, when

414 compared to control HeLa-NT cells ${ }^{32}$. However, whether there is a feedback loop mechanism

415 between glycolysis and DNA repair pathway and its potential role in NHEJ will require further

416 investigation.

418 Depletion of another DNA repair enzyme, DNA-PKc is previously shown to cause alteration in 419 metabolic pathway and TP53 levels ${ }^{33}$. Enhanced p53 activity is a critical cellular signal for DNA 420 damage. Under normal condition, p53 remains in an inactivated state. However, in response to 421 DNA damage, the p53 is activated and drives transcription of factors involved in the apoptosis, 422 cell cycle, DNA repair, and cellular senescence ${ }^{34,35}$. Therefore, to compensate for defective 423 XRCC4 and MRE11, our results show that the metabolic and p53 pathways are altered as an 424 adaption to cellular stress introduced by DSB and in absence of efficient NHEJ repair systems.

426 A protein-protein interaction network analysis suggest interconnectivity between the 427 transcriptional responses for each NHEJ group (Fig 6A). This is consistent with our hypothesis of 428 cross talk between different NHEJ pathways. While the previously discussed p53 response has an 429 well-known connection with DNA damage and repair, chromatin modifications may be required 430 to give NHEJ repair proteins access to the DSB. One major DEG category supports a possible role 
431 for chromatin modification as part of the regulator response to blocking NHEJ. A set of histones

432 genes that were identified in the protein-protein interaction studies, and these genes are also part

433 of Alcoholism pathway that is highly enriched in the XRCC4(-/-) cells treated with Mirin (Fig.

434 6B). HDACs deacetylation histones have a major influence on chromatin structure and regulates

435 the activation of DNA repair proteins ${ }^{36}$. Therefore, the DEG for these pathways was not surprising.

436 There are several reports supporting the profiles of histone acetylation affecting NHEJ ${ }^{37-40}$.

437 HDAC inhibitors impair DNA repair, suggesting that upregulation of HDAC deacetylation 438 contributes to DNA repair in Mirin treated XRCC4(-/-) cells ${ }^{41}$.

440 In conclusion, our results demonstrate that NHEJ is robust, even when key proteins thought to be 441 essential for the two known NHEJ pathways are blocked. Surprisingly, the majority of NHEJ 442 activity was preserved with a corresponding transcriptional response. These results are an 443 important step forward in identifying regulatory interactions between NHEJ pathways.

\section{Authors contribution}

$446 \mathrm{RB}$ and MRS conceived the hypotheses and experiments. ST helped collect and analyze literature 447 editing data. RB, AB conducted the experiments and analyzed the data, LB and DE helped with 448 sequencing of $X R C C 4(-/-)$ cells, $\mathrm{CG}$ and XW collected and processed the RNA-seq raw data, RB, $449 \mathrm{AB}$ and MRS contributed to writing of the manuscript and MRS provided funding and space for 450 the work.

\section{Conflict of interest}

453 The authors declare that there are no conflicts of interest. 


\section{$455 \quad$ Funding}

456 The Nevada Governor's office of Economic Development and by the National Institutes of Health 457 (R56 AI109156 and P20 GM121325 to MRS) funded this research. This research is also supported 458 by the Prabhu Endowed Professorship to MRS.

\section{Acknowledgements}

461 Flow cytometry and initial RNA-seq data analyses were performed with resources from the

462 Nevada Institute of Personalized Medicine (NIPM) and the National Supercomputing Institute at 463 University of Nevada Las Vegas (UNLV). The authors would like to acknowledge Edwin Oh and 464 Shirley Shen and Genome Acquisition and Analysis (GAA) Core from the NIH Center of 465 Biomedical Research Excellence (COBRE) at NIPM. Real time PCR was performed at UNLV 466 genomics core facility and is acknowledged.

\section{References}

469 1. Hakem, R. DNA-damage repair; the good, the bad, and the ugly. EMBO J 27, 589-605 $470 \quad$ (2008).

471 2. Lindahl, T. Instability and decay of the primary structure of DNA. Nature 362, 709-715 $472 \quad$ (1993).

473 3. Peterson, C. L. \& Côté, J. Cellular machineries for chromosomal DNA repair. Genes Dev. $474 \quad 18,602-616(2004)$.

475 4. Mao, Z., Bozzella, M., Seluanov, A. \& Gorbunova, V. Comparison of nonhomologous end 476 joining and homologous recombination in human cells. DNA Repair (Amst) 7, 1765-1771 477 (2008). 
478 5. Mao, Z., Bozzella, M., Seluanov, A. \& Gorbunova, V. Comparison of nonhomologous end 479 joining and homologous recombination in human cells. DNA Repair (Amst) 7, 1765-1771 $480 \quad$ (2008).

481 6. Rodgers, K. \& McVey, M. Error-prone repair of DNA double-strand breaks. J Cell Physiol $482 \quad 231,15-24(2016)$.

483 7. Sishc, B. J. \& Davis, A. J. The Role of the Core Non-Homologous End Joining Factors in 484 Carcinogenesis and Cancer. Cancers (Basel) 9, (2017).

485 8. Kabotyanski, E. B., Gomelsky, L., Han, J.-O., Roth, D. B. \& Stamato, T. D. Double-strand 486 break repair in Ku86- and XRCC4-deficient cells. Nucleic Acids Research 26, 5333-5342 $487 \quad$ (1998).

488 9. Deriano, L. \& Roth, D. B. Modernizing the nonhomologous end-joining repertoire: 489 alternative and classical NHEJ share the stage. Annu Rev Genet 47, 433-455 (2013).

490 10. Trimidal, S. G. et al. Can Designer Indels Be Tailored by Gene Editing? Bioessays 41, $491 \quad$ e1900126 (2019).

492 11. Schulte-Uentrop, L., El-Awady, R. A., Schliecker, L., Willers, H. \& Dahm-Daphi, J. Distinct 493 roles of XRCC4 and Ku80 in non-homologous end-joining of endonuclease- and ionizing 494 radiation-induced DNA double-strand breaks. Nucleic Acids Res 36, 2561-2569 (2008).

495 12. Uziel, T. et al. Requirement of the MRN complex for ATM activation by DNA damage. 496 EMBO J 22, 5612-5621(2003).

497 13. Zha, S., Boboila, C. \& Alt, F. W. Mre11: roles in DNA repair beyond homologous 498 recombination. Nat Struct Mol Biol 16, 798-800 (2009).

499 14. Stracker, T. H. \& Petrini, J. H. J. The MRE11 complex: starting from the ends. Nat Rev Mol $500 \quad$ Cell Biol 12, 90-103 (2011). 
501 15. Shibata, A. et al. DNA double-strand break repair pathway choice is directed by distinct

502 MRE11 nuclease activities. Mol Cell 53, 7-18 (2014).

503 16. Xie, A., Kwok, A. \& Scully, R. Role of mammalian Mre11 in classical and alternative

504 nonhomologous end joining. Nat Struct Mol Biol 16, 814-818 (2009).

505 17. Miller, J. C. et al. A TALE nuclease architecture for efficient genome editing. Nat.

$506 \quad$ Biotechnol. 29, 143-148 (2011).

507 18. Strong, C. L. et al. Damaging the Integrated HIV Proviral DNA with TALENs. PLoS ONE

$508 \quad \mathbf{1 0}, \mathrm{e} 0125652(2015)$.

509 19. Ewing, B., Hillier, L., Wendl, M. C. \& Green, P. Base-calling of automated sequencer traces

510 using phred. I. Accuracy assessment. Genome Res. 8, 175-185 (1998).

511 20. Metsalu, T. \& Vilo, J. ClustVis: a web tool for visualizing clustering of multivariate data

512 using Principal Component Analysis and heatmap. Nucleic Acids Research 43, W566-W570

$513 \quad$ (2015).

514 21. Blighe, K. kevinblighe/EnhancedVolcano. (2020).

515 22. Zhou, Y. et al. Metascape provides a biologist-oriented resource for the analysis of systems-

$516 \quad$ level datasets. Nature Communications 10, 1523 (2019).

517 23. Miller, K. M. et al. Human HDAC1 and HDAC2 function in the DNA-damage response to

518 promote DNA nonhomologous end-joining. Nature Structural \& Molecular Biology 17,

$519 \quad 1144-1151(2010)$.

520 24. Xing, M. et al. Interactome analysis identifies a new paralogue of XRCC4 in non-

521 homologous end joining DNA repair pathway. Nature Communications 6, 6233 (2015).

522 25. Daley, J. M. \& Sung, P. 53BP1, BRCA1, and the Choice between Recombination and End

523 Joining at DNA Double-Strand Breaks. Mol Cell Biol 34, 1380-1388 (2014). 
524 26. Shrivastav, M., De Haro, L. P. \& Nickoloff, J. A. Regulation of DNA double-strand break 525 repair pathway choice. Cell Research 18, 134-147 (2008).

526 27. Xia, W. et al. Two-way crosstalk between BER and c-NHEJ repair pathway is mediated by $527 \quad$ Pol- $\beta$ and Ku70. FASEB J 33, 11668-11681 (2019).

528 28. Shamanna, R. A. et al. WRN regulates pathway choice between classical and alternative 529 non-homologous end joining. Nat Commun 7, (2016).

530 29. Hristova, D. B., Lauer, K. B. \& Ferguson, B. J. Viral interactions with non-homologous end531 joining: a game of hide-and-seek. Journal of General Virology, 101, 1133-1144 (2020).

532 30. Sharma, V. et al. Oxidative stress at low levels can induce clustered DNA lesions leading to 533 NHEJ mediated mutations. Oncotarget 7, 25377-25390 (2016).

534 31. Huangyang, P. \& Simon, M. C. Hidden features: exploring the non-canonical functions of 535 metabolic enzymes. Dis Model Mech 11, (2018).

536 32. Bhatt, A. N. et al. Transient elevation of glycolysis confers radio-resistance by facilitating 537 DNA repair in cells. BMC Cancer 15, 335 (2015).

538 33. Ali, S. I., Najaf-Panah, M. J., Sena, J., Schilkey, F. D. \& Ashley, A. K. Comparative gene 539 expression in cells competent in or lacking DNA-PKcs kinase activity following etoposide 540 exposure reveal differences in gene expression associated with histone modifications, 541 inflammation, cell cycle regulation, Wnt signaling, and differentiation. bioRxiv 2020.09.17.300129 (2020) doi:10.1101/2020.09.17.300129.

543 34. Nd, L. \& Sp, J. Regulation of p53 in response to DNA damage. Oncogene vol. 18 544 https://pubmed.ncbi.nlm.nih.gov/10618704/ (1999).

545 35. Mijit, M., Caracciolo, V., Melillo, A., Amicarelli, F. \& Giordano, A. Role of p53 in the 546 Regulation of Cellular Senescence. Biomolecules 10, (2020). 
547 36. Piekna-Przybylska, D., Bambara, R. A. \& Balakrishnan, L. Acetylation regulates DNA

548 repair mechanisms in human cells. Cell Cycle 15, 1506-1517 (2016).

549 37. Tamburini, B. A. \& Tyler, J. K. Localized histone acetylation and deacetylation triggered by

550 the homologous recombination pathway of double-strand DNA repair. Mol Cell Biol 25,

$551 \quad 4903-4913(2005)$.

552 38. Jazayeri, A., McAinsh, A. D. \& Jackson, S. P. Saccharomyces cerevisiae Sin3p facilitates

553 DNA double-strand break repair. Proc Natl Acad Sci U S A 101, 1644-1649 (2004).

554 39. Martin, S. G., Laroche, T., Suka, N., Grunstein, M. \& Gasser, S. M. Relocalization of 555 telomeric Ku and SIR proteins in response to DNA strand breaks in yeast. Cell 97, 621-633

$556 \quad(1999)$.

557 40. Mills, K. D., Sinclair, D. A. \& Guarente, L. MEC1-dependent redistribution of the Sir3

558 silencing protein from telomeres to DNA double-strand breaks. Cell 97, 609-620 (1999).

559 41. Munshi, A. et al. Histone deacetylase inhibitors radiosensitize human melanoma cells by

560 suppressing DNA repair activity. Clin Cancer Res 11, 4912-4922 (2005). 


\section{$571 \quad$ Figure legends:}

\section{Figure 1: Construction and validation of NHEJ reporter system}

573 A) Schematic representation of NHEJ reporter system and plausible outcome when both NHEJ 574 pathways are blocked, where TBS $=$ TALEN binding site; $\mathrm{CMV}=$ cytomegalovirus promoter. $\mathrm{B}$ )

575 Fluorescent microscopy validation of reporter system. C) Western blot confirmation of XRCC4 (-

576 /-) clonal selection in HeLa cells where 2G3 clone had no expression. D) Sequence of region of 577 XRCC4 genomic DNA alleles for wild type cells and XRCC4(-/-) 2G3 clone. Deletions are 578 indicated by “-”. 2G3 clone alleles have a frameshift indel, thus are a biallelic knockout. F) MTT 579 assay of HeLa-NT cells treated with $100 \mathrm{mM}$ of Mirin when compared to HeLa-NT cells. 580 Statistically significant differences were determined by an ANOVA analysis where *denotes $581 \mathrm{p}<0.05$.

583 Figure 2: DNA double stand break repair when both XRCC4 and MRE-11 are blocked.

584 Representative fluorescence microscopy images of NHEJ editing when C-NHEJ and A-NHEJ are 585 blocked. HeLa-NT (A-D) and XRCC4(-/-) (E-H). Cells co-transfected with TALEN reporter 586 plasmid (expressing mCherry) along with either empty vector or TALEN plasmids where GFP ${ }^{+}$ 587 cells indicate DNA repair events and treated with 100uM Mirin wherever indicated. Cells were 588 analyzed 48 hrs post-transfection and representative images are shown. TALEN reporter with 589 empty vector (A, E), empty vector with Mirin (B, F), TALEN plasmid (C, G), TALEN plasmid 590 with Mirin (D, H) are shown.

592 Figure 3: Measurement of NHEJ efficiency using flow cytometry. 
593 A) Representative images of cells positive for mCherry expression followed by $\mathrm{GFP}^{+}$expression.

594 B) Mean $\mathrm{GFP}^{+}$cells were plotted. Experiments were done in triplicates and data was analysed 595 using FLowJo 10.7.1. Statistical significance was determined by ANOVA where*indicates $\mathrm{p}<0.01$

597 Figure 4: Principal component analysis (PCA), Venn Diagram and Heatmap from RNA-seq 598 profile.

599 A) A PCA plot for HeLa-NT, XRCC4(-/-), Mirin and XRCC4(-/-)+ Mirin from whole600 transcriptome RNA-seq data using ClusVis ${ }^{20}$. B) A Venn diagram representing shared and unique 601 DEGs across 3 categories (i) XRCC4(-/-) (blue) (iii) Mirin treated (green) (iii) XRCC4(-/-) with 602 Mirin treated (red) when compared to HeLa-NT cells. C) A heatmap of top DEGs across each 603 sample categories.

605 Figure 5: Functional enrichment analysis

606 A) A heatmap with embedded dendogram showing relationships between enriched GO/KEGG 607 terms and canonical pathways. 0.3 kappa score was applied as the threshold to cast the tree into 608 term clusters.

609 B) A network graph with enrichment ontology clusters colored by cluster ID. Each term is 610 represented by a node, where its size is proportional to the number of genes for each term. Terms 611 with a similarity score $>0.3$ are linked by an edge (the thickness of the edge represents the 612 similarity score). The network was created with Cytoscape (v3.1.2).

\section{Figure 6: Protein-protein interaction network analysis.}

615 A) PPI network of DEGs across all three NHEJ categories. Nodes are displayed as pies to indicate 616 NHEJ sample. B) MCODE components were identified from merged network for all samples. 
617 Each MCODE network is assigned a unique color and the network was generated with Cytoscape

618 (v3.1.2). C) MCODE GO term, description and $\mathrm{p}$ values are shown.

620 Supplementary Figure 1: Validation of RNA-seq profile across all the categories.

621 Changes in gene expression determined by real time PCR. The data were represented as mean \pm $622 \mathrm{SD}$ and significance between groups were determined by ANOVA test where denoted $p<0.05$ 623 and NS=not significant.

625 Supplementary Figure S2: Volcano chart and functional enrichment analysis.

626 Volcano chart showing differentially expressed genes with $\mathrm{FDR}<0.01$ and $\log \mathrm{FC}>=1.2$ compared

627 to HeLa-NT cells A) XRCC4(-/-), B) Mirin treatment, C) XRCC4(-/-) with Mirin treatment. 


\section{Figures}

A

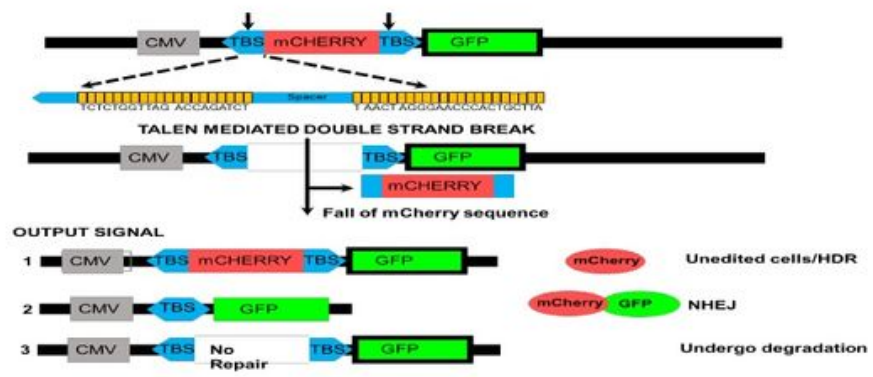

B

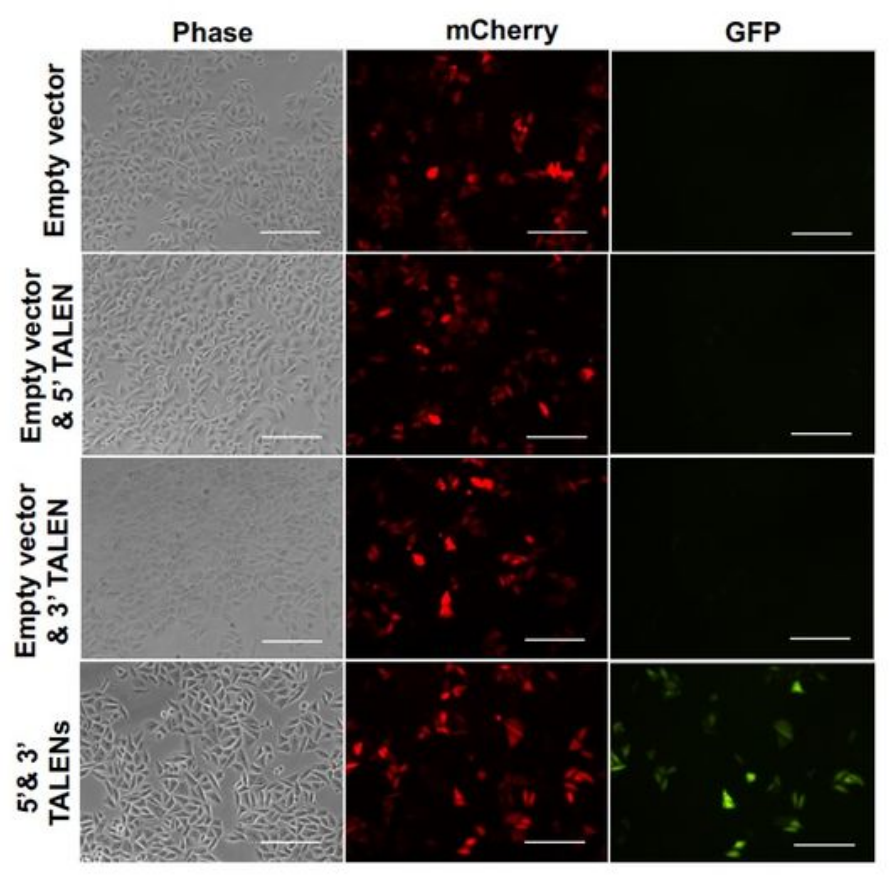

C

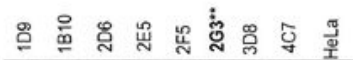
$\mathrm{XRCC} 4$
$55 \mathrm{KDa}$

D cagrccatgctganatgaccatcagraagtgraat Hela gscrRna CAGTC--TGCIGAATGACCATCAGTAAGTGTAAT 2 G3 gXRCCARNA a-1 CAGTC-------GACCATCAGTAAGTGTAAT 2 G3 gXRCC4RNA a-2
E

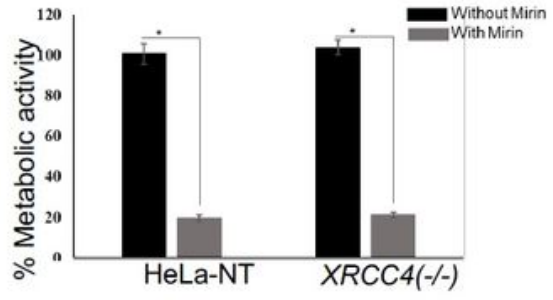

Figure 1

Construction and validation of NHEJ reporter system A) Schematic representation of NHEJ reporter system and plausible outcome when both NHEJ pathways are blocked, where TBS = TALEN binding site; $\mathrm{CMV}=$ cytomegalovirus promoter. B) Fluorescent microscopy validation of reporter system. C) Western 
blot confirmation of XRCC4 (-/-) clonal selection in HeLa cells where $2 \mathrm{G} 3$ clone had no expression. D) Sequence of region of XRCC4 genomic DNA alleles for wild type cells and XRCC4(-/-) $2 \mathrm{G} 3$ clone. Deletions are indicated by "-". 2G3 clone alleles have a frameshift indel, thus are a biallelic knockout. F) MTT assay of HeLa-NT cells treated with $100 \mathrm{mM}$ of Mirin when compared to HeLa-NT cells. Statistically significant differences were determined by an ANOVA analysis where *denotes $p<0.05$.
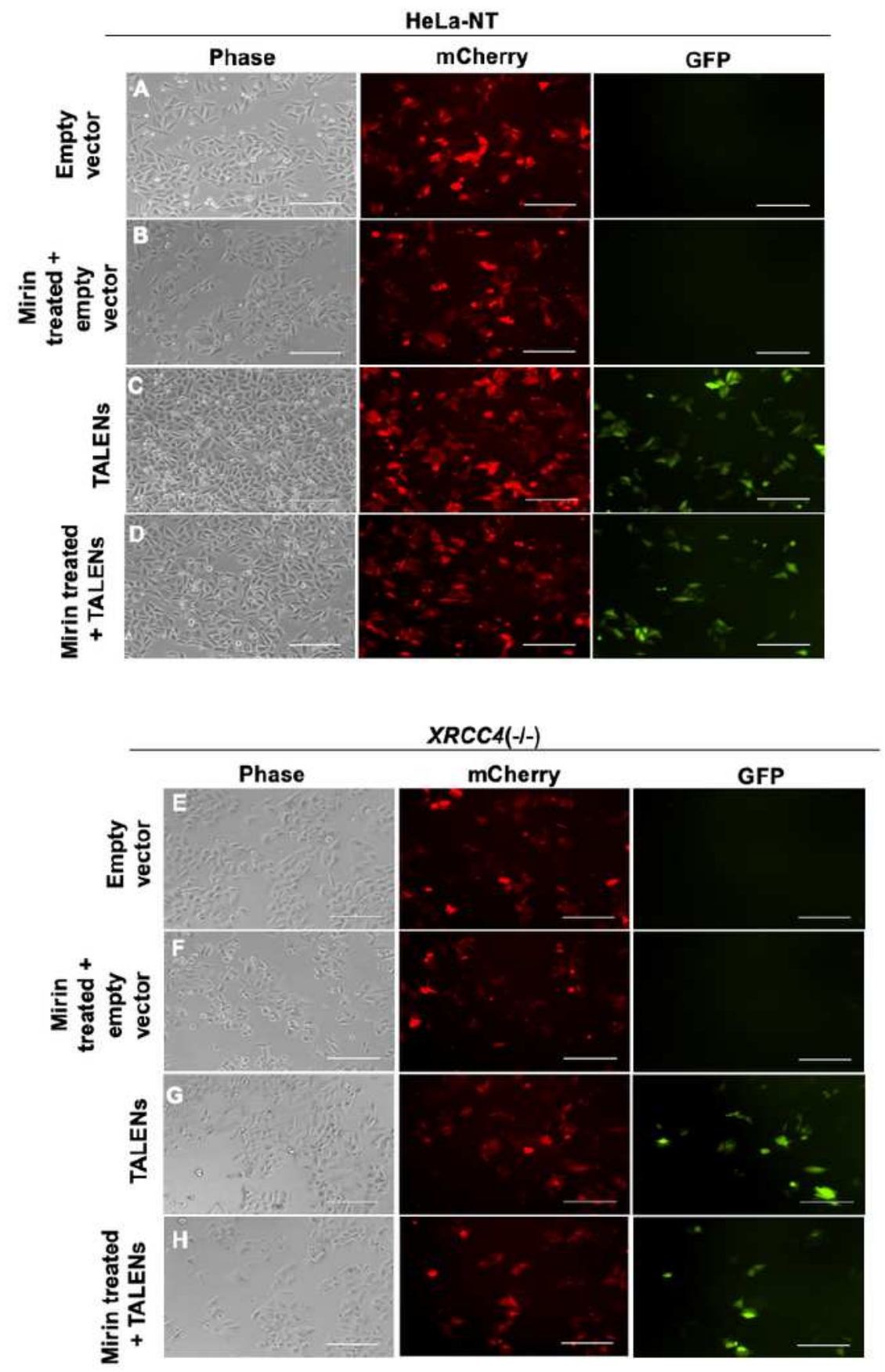

Figure 2 
DNA double stand break repair when both XRCC4 and MRE-11 are blocked. Representative fluorescence microscopy images of NHEJ editing when C-NHEJ and A-NHEJ are blocked. HeLa-NT (A-D) and XRCC4(-/-) (E-H). Cells co-transfected with TALEN reporter plasmid (expressing mCherry) along with either empty vector or TALEN plasmids where GFP+ cells indicate DNA repair events and treated with $100 \mathrm{uM}$ Mirin wherever indicated. Cells were analyzed $48 \mathrm{hrs}$ post-transfection and representative images are shown. TALEN reporter with empty vector (A, E), empty vector with Mirin (B, F), TALEN plasmid (C, G), TALEN plasmid with Mirin $(D, H)$ are shown.

A

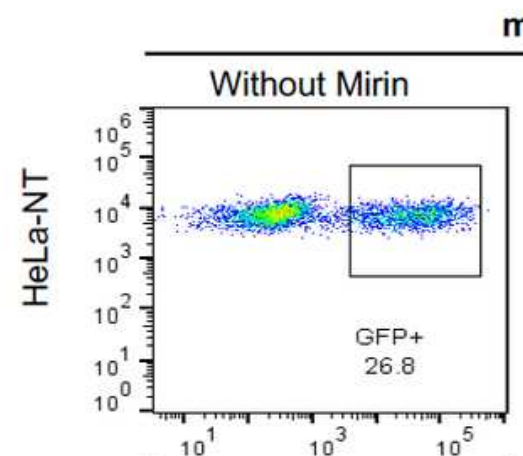

mCherry+
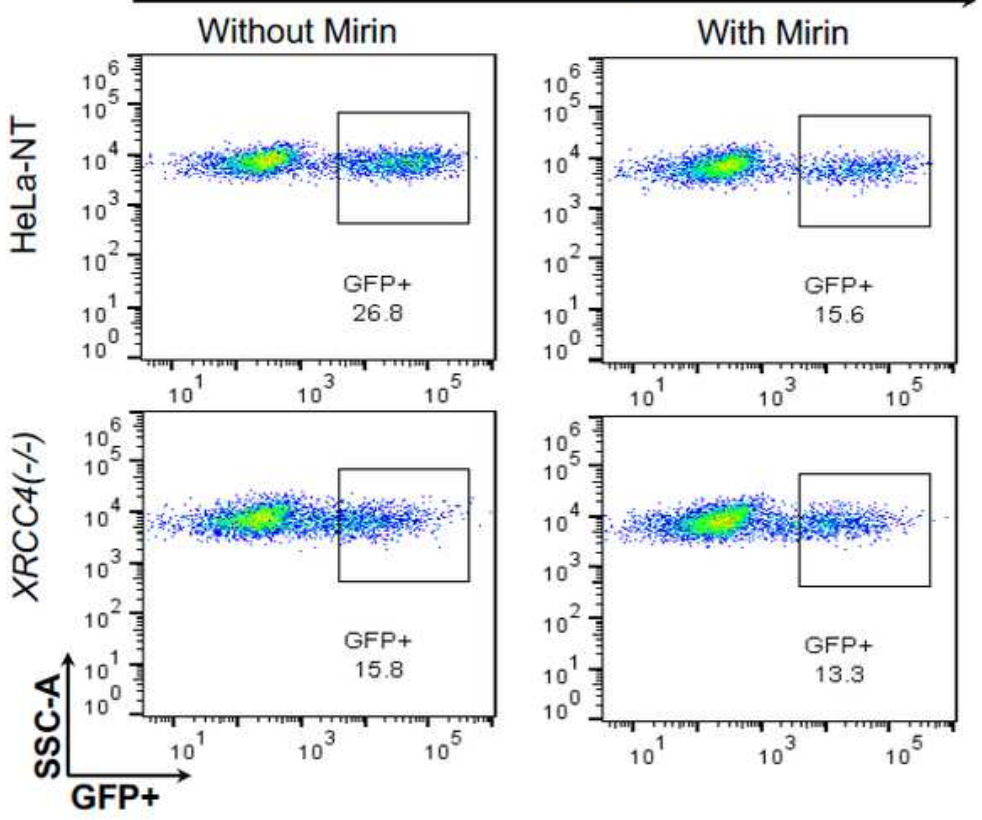

B

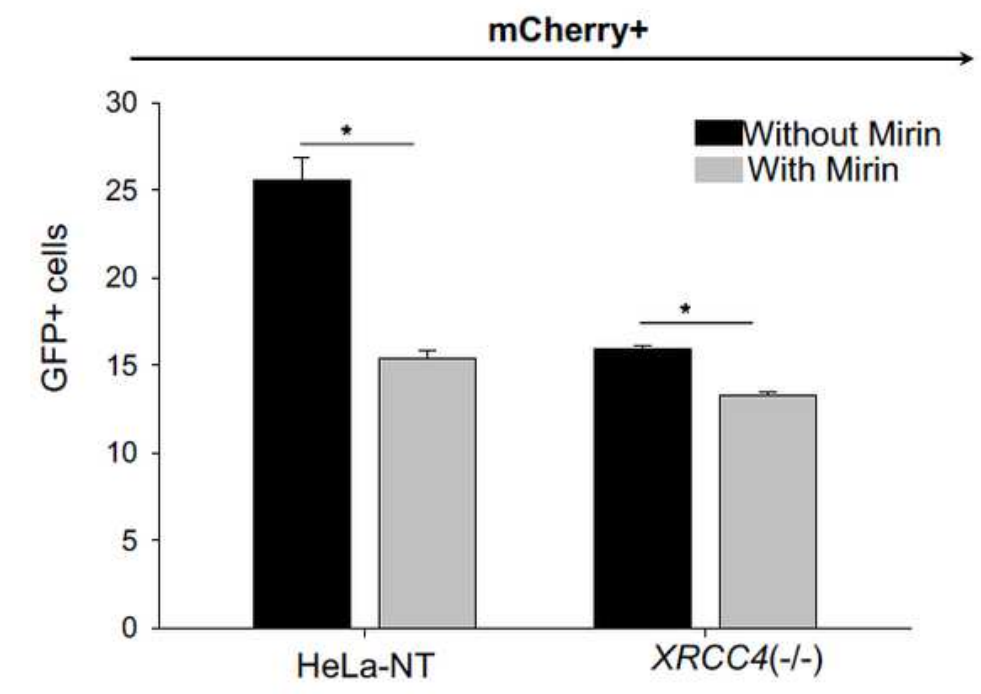




\section{Figure 3}

Measurement of NHEJ efficiency using flow cytometry. A) Representative images of cells positive for mCherry expression followed by GFP+ expression. B) Mean GFP+ cells were plotted. Experiments were done in triplicates and data was analysed using FLowJo 10.7.1. Statistical significance was determined

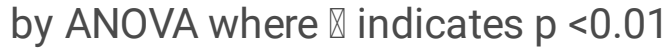

\section{Fig. 4}

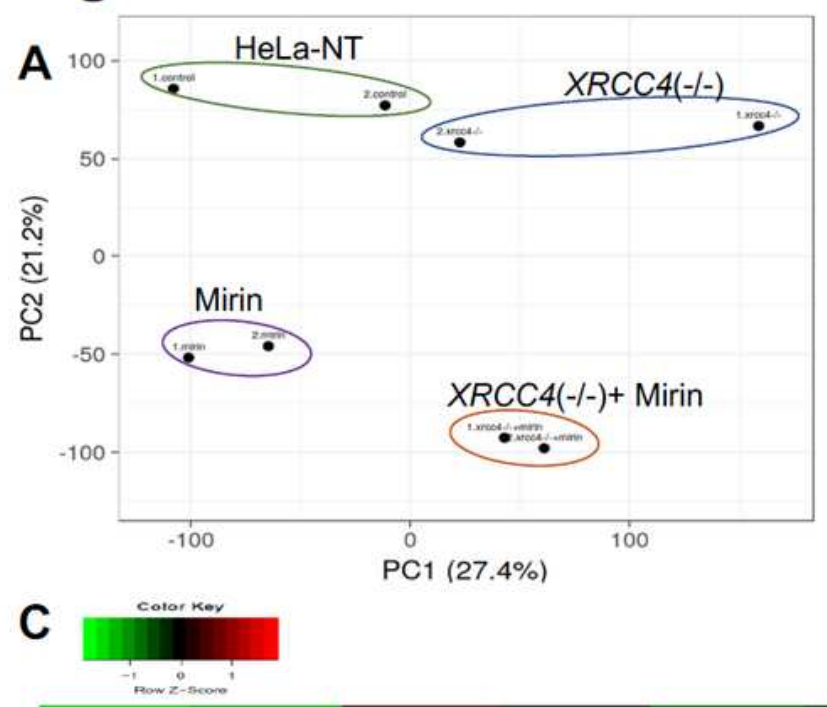

B

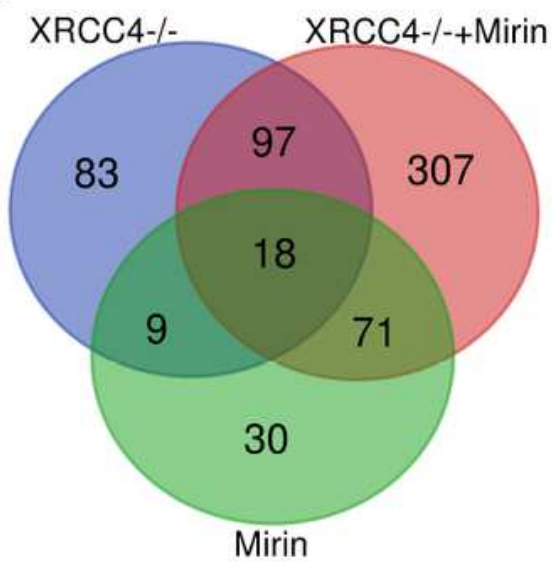

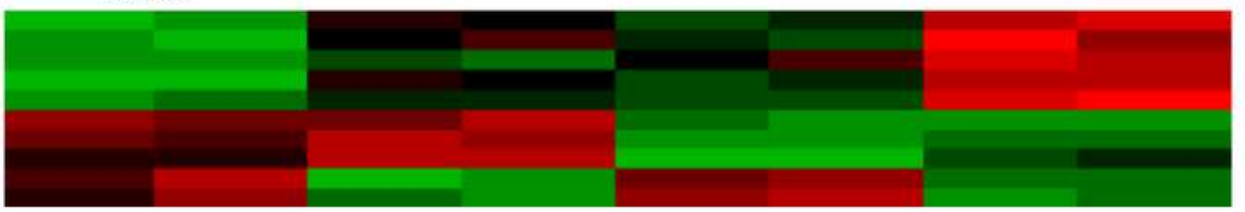

CCND1
SLC7A11

$R P(22$

HIST1 $2 A C$ Hististin COL 15

ISG 20
OASL

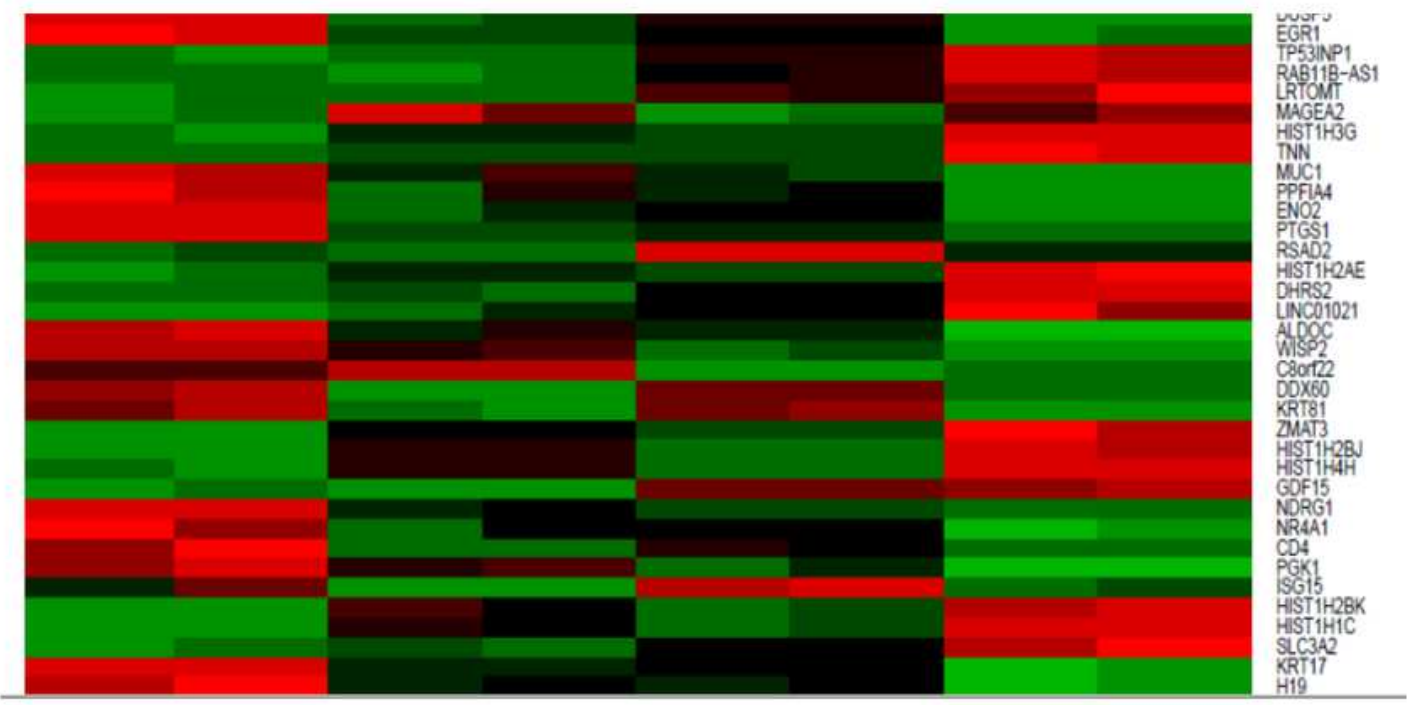


Principal component analysis (PCA), Venn Diagram and Heatmap from RNA-seq profile. A) A PCA plot for HeLa-NT, XRCC4(-/-), Mirin and XRCC4(-/-)+ Mirin from whole transcriptome RNA-seq data using ClusVis 20. B) A Venn diagram representing shared and unique DEGs across 3 categories (i) XRCC4(-/-) (blue) (iii) Mirin treated (green) (iii) XRCC4(-/-) with Mirin treated (red) when compared to HeLa-NT cells. C) A heatmap of top DEGs across each sample categories.
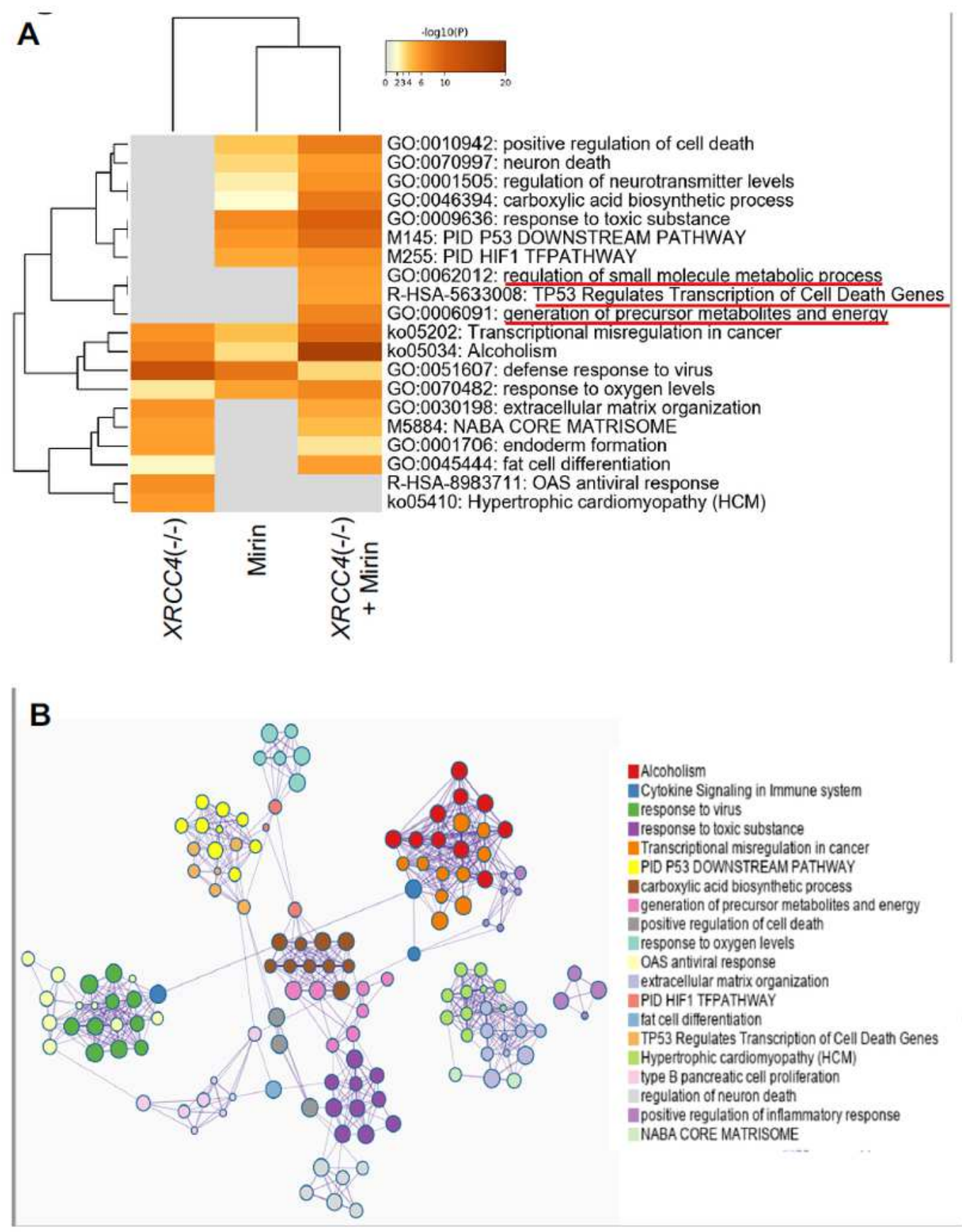
Functional enrichment analysis A) A heatmap with embedded dendogram showing relationships between enriched GO/KEGG terms and canonical pathways. 0.3 kappa score was applied as the threshold to cast the tree into term clusters. B) A network graph with enrichment ontology clusters colored by cluster ID. Each term is represented by a node, where its size is proportional to the number of genes for each term. Terms with a similarity score $>0.3$ are linked by an edge (the thickness of the edge represents the similarity score). The network was created with Cytoscape (v3.1.2).
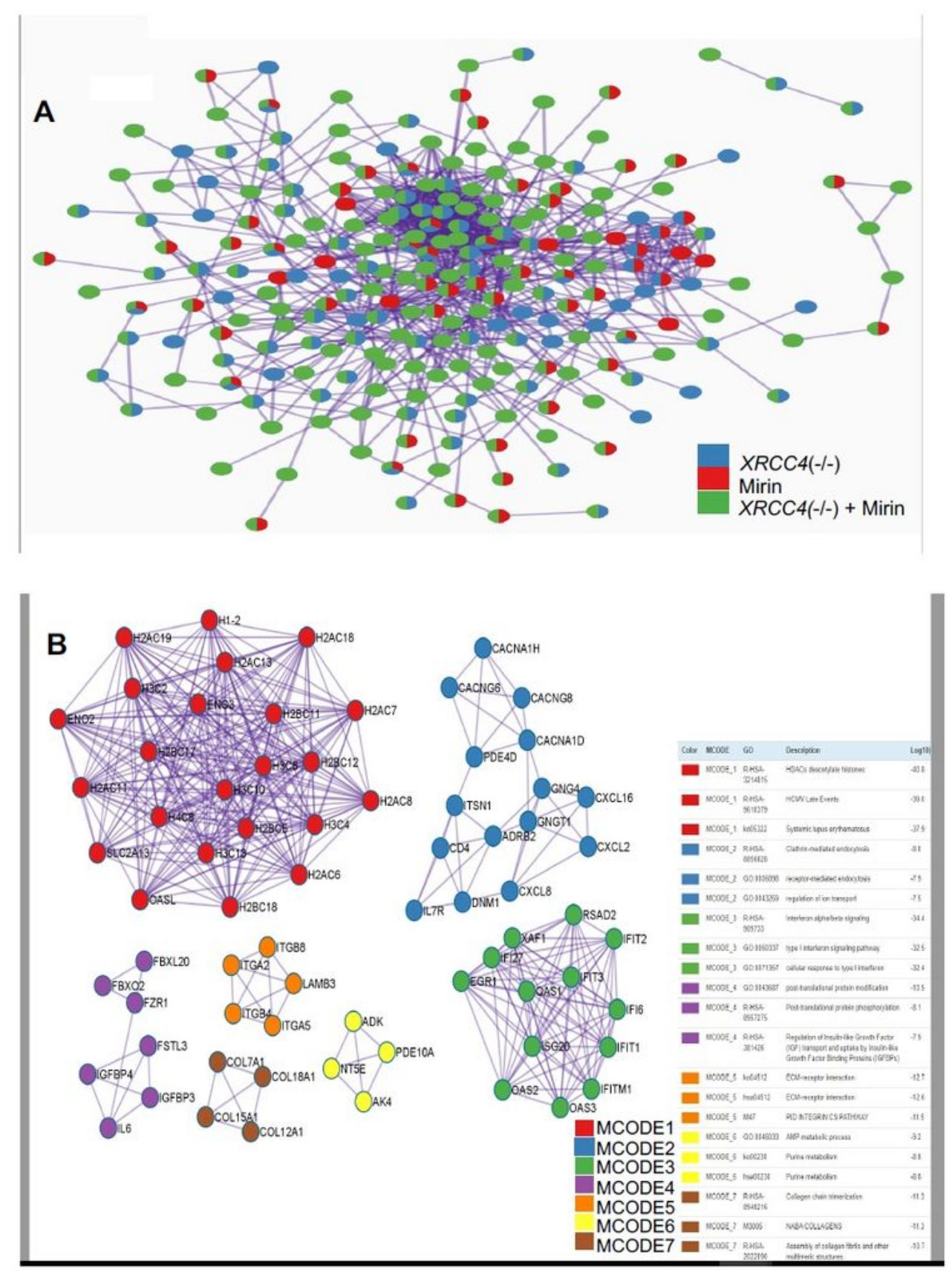
Protein-protein interaction network analysis. A) PPI network of DEGs across all three NHEJ categories. Nodes are displayed as pies to indicate NHEJ sample. B) MCODE components were identified from merged network for all samples. Each MCODE network is assigned a unique color and the network was generated with Cytoscape (v3.1.2). C) MCODE GO term, description and $p$ values are shown.

\section{Supplementary Files}

This is a list of supplementary files associated with this preprint. Click to download.

- NHEJsuppfigures4.24.21.pdf

- SupplementaryTable1.xlsx

- SupplementaryTable2.xlsx 